\section{Molecular Detection and Characterization of Rickettsia asembonensis in Human Blood, Zambia}

\author{
Lavel C. Moonga, Kyoko Hayashida, \\ Namwiinga R. Mulunda, Yukiko Nakamura, James \\ Chipeta, ${ }^{1}$ Hawela B. Moonga, \\ Boniface Namangala, Chihiro Sugimoto, \\ Zephaniah Mtonga, Mable Mutengo, Junya Yamagishi
}

Author affiliations: Hokkaido University, Sapporo, Japan (L.C. Moonga, K. Hayashida, Y. Nakamura, C. Sugimoto,

J. Yamagishi); University of Zambia, Lusaka, Zambia

(N.R. Mulunda, J. Chipeta, B. Namangala, M. Mutengo); National Malaria Control Center, Lusaka (H.B. Moonga); Chongwe District Hospital, Lusaka (Z. Mtonga); Levy Mwanawasa Medical University, Lusaka (M. Mutengo)

DOI: https://doi.org/10.3201/eid2708.203467

Rickettsia asembonensis is a flea-related Rickettsia with unknown pathogenicity to humans. We detected $R$. asembonensis DNA in 2 of 1,153 human blood samples in Zambia. Our findings suggest the possibility of $R$. asembonensis infection in humans despite its unknown pathogenicity.

$R$ ickettsia asembonensis is a fleaborne rickettsia closely related to Rickettsia felis and is thus referred to as an $R$. felis-like organism. R. asembonensis was first detected in cat fleas in Kenya and subsequently reported worldwide $(1,2)$. Although $R$. felis has been increasingly recognized as a human infective agent that can cause human febrile disease, the infectivity and pathogenicity of $R$. asembonensis in humans is largely unknown. Recent investigations in patients with febrile illness and petechial lesions identified R. asembonensis DNA and antibodies for rickettsial antigens in Malaysia $(3,4)$. Furthermore, $R$. asembonensis was isolated in cellular cultures from patients in Peru with acute febrile illness and confirmed by sequencing (5). These reports suggest the possibility of $R$. asembonensis as a human infective agent. However, no direct evidence of $R$. felis and $R$. asembonensis as an etiologic agent of human illness has been established. A previous study in Zambia revealed the predominant existence of R. asembonensis and R. felis in cat fleas (6). Our study investigates the presence of these rickettsiae in human blood in Zambia.

We obtained 753 residual patient blood samples from hospitals in urban Lusaka $(n=519)$ and the

${ }^{1}$ Deceased.
Chongwe District $(\mathrm{n}=234)$ of Zambia. Approximately half of the samples $(303 / 753)$ were traceable to clinical records of patients. The common clinical conditions among those patients included fever, anemia, meningitis, septicemia, and sickle cell anemia (Appendix Table 1, https://wwwnc.cdc.gov/EID/ article/27/8/20-3467-App1.pdf). In addition, we obtained dried blood spots on Whatman FTA classic cards (Millipore Sigma, https://www.sigmaaldrich. com) from healthy volunteers from rural eastern $(\mathrm{n}=$ $200)$ and central $(n=200)$ provinces to assess rickettsia infection in healthy rural persons. The study was approved by the National Health Research Authority of Zambia through the Biomedical Research Ethics Committee (reference no. 007-10-18).

We extracted genomic DNA and subjected it to PCR screening that targeted the citrate synthase gene ( $g l t A$ ) of Rickettsia. We subjected the positive samples to multiple-gene sequencing analysis targeting the 17$\mathrm{kDa}$ common antigen $(h t r A)$, outer membrane protein A $(O m p A)$, and outer membrane protein B $(O m p B)$ genes using previously described primers (Appendix Table 2). We aligned the sequences using MAFFT (https://mafft.cbrc.jp/alignment/server) and performed phylogenetic analysis by the neighbor-joining method using MEGA7 (https:/ / www.megasoftware. net). We determined the estimated Rickettsia bacterial burden in Rickettsia-positive blood samples by OmpA quantitative PCR by using published primers. We further testsed the gltA PCR-positive samples for malaria by nested PCR (Appendix Table 2).

We detected $R$. asembonensis in $0.39 \%(2 / 519)$ samples from the urban Lusaka District by gltA PCR. The samples from the Chongwe District and the rural areas of the eastern and central provinces were all negative, although the possibility that dried blood spot samples from rural areas might have lower detection sensitivity cannot be ruled out. BLAST analysis (https:/ / blast.ncbi.nlm.nih.gov/Blast.cgi) of the gltA sequences obtained (GenBank accession nos. LC557154 and LC557155) showed 100\% homology to R. asembonensis identified in cat fleas from human dwellings and domestic dogs in 3 countries: Senegal (GenBank accession no. JF966774), Kenya (accession no. JN315968), and Zambia (accession no. LC431490) $(6,7)$. Comparing the sequenced gltA with those detected in fleas from Peru (GenBank accession no. KY650697) and other regions in the Americas showed 99.8\% similarity. Phylogenetic analysis of gltA confirmed the detected sequences' closer relatedness by clustering with genes from cat fleas from sub-Saharan Africa, a distinct cluster from other regions (Figure). The $O m p A, O m p B$, and $h$ tr $A$ sequences showed clustering without regional 


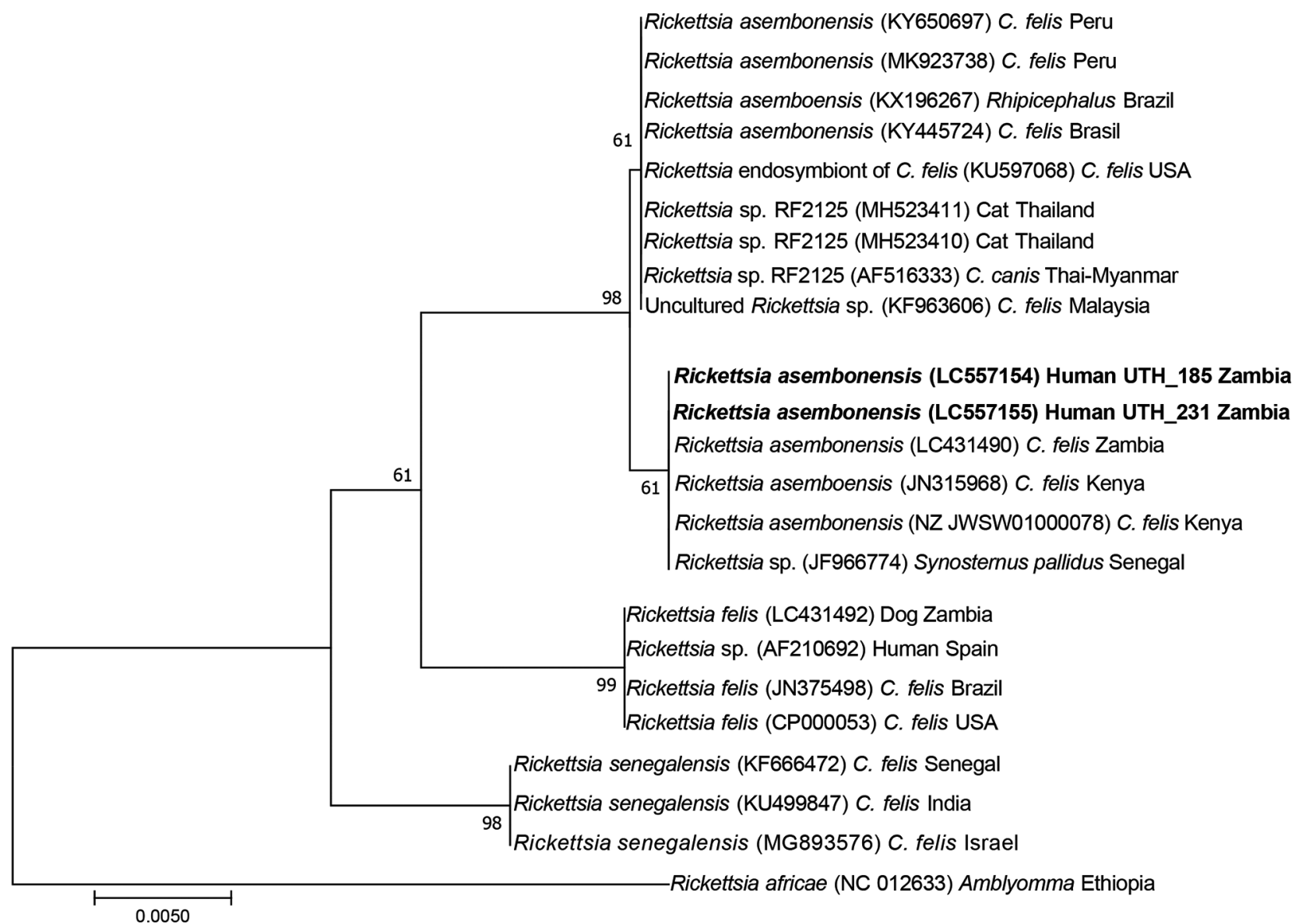

Figure. Phylogenetic tree of Rickettsia felis and R. felis-like organisms based on the sequences of the gltA gene (581 bp) from human blood samples collected from Zambia, 2019 (in bold). The tree was constructed using the neighbor-joining method with the maximumlikelihood model. Bootstrap values are shown on nodes based on 1,000 replicates. Sequences are identified by species name, GenBank accession number, host, and country of detection. Scale bar indicates nucleotide substitutions per site.

discrimination (data not shown). The obtained nucleotides are available in GenBank under accession nos. LC557154-61. Detection of genotypically similar R. asembonensis in persons and cat fleas in Zambia suggests possible human infection by $R$. asembonensis through cat flea bites. Nevertheless, the epidemiologic cycle and pathogenicity of $R$. asembonensis and other related $R$. felis-like organisms remain to be elucidated.

The patient identified as UTH_185 in whom $R$. asembonensis was detected had a medical record of anemia and weight loss (Table). The malaria test was negative. Despite the limited association of Rickettsia infection with anemia, severe R. felis infection has been reported with severe anemia, possibly attributable to hemorrhage from vascular damage in rickettsial disease (8). However, the observed evidence was limited and could not establish $R$. asembonensis as the cause of these symptoms. The second $R$. asembonensis-positive sample from the patient identified as UTH_231 had limited clinical information, which did not allow for further interpretation. The $2 R$. asembonensis-positive blood samples showed estimated

\begin{tabular}{lcc}
\hline $\begin{array}{l}\text { Table. Selected demographic and clinical characteristics of } 2 \text { persons in whom Rickettsia asembonensis was detected from blood } \\
\text { samples collected in Zambia }\end{array}$ & \\
\hline Characteristic & Patient UTH_185 & Patient UTH_231 \\
\hline Age, y & 42 & 45 \\
Sex & Female & Female \\
Residential area & Lusaka & Lusaka \\
Clinical manifestation & Anemia and weight loss & No information \\
Estimated rickettsia genome copies $/ \mathrm{mL}$ blood & 890,000 & $2,150,000$ \\
Malaria test & Negative & Negative \\
\hline
\end{tabular}


DNA quantities of 890,000 copies / $\mathrm{mL}$ of blood from patient UTH_185 and 2,100,000 copies/mL of blood from patient UTH_231 (Table). These results are within the same range as a previous study for Rickettsia rickettsii estimated rickettsial burden (9).

In conclusion, detection of $R$. asembonensis of identical genotype in cat fleas and human blood in Zambia suggests possible transmission from cat fleas to humans. Given the worldwide distribution of $R$. asembonensis, further studies to elucidate its pathogenicity and epidemiologic cycle are warranted.

This study was supported by the Japan Agency for Medical Research and Development (grant no.

JP21wm0125008). The funder had no role in the design of the study, data collection, analysis, decision to publish, or preparation of the manuscript. This work is dedicated to the memory of James Chipeta. May his soul rest in peace.

\section{About the Author}

Dr. Moonga is a postdoctoral research fellow at Hokkaido University. His research interests include arthropodborne zoonoses, specifically fleaborne rickettsia infections as a possible cause of febrile illness, and developing of rapid molecular diagnostic tools.

\section{References}

1. Jiang J, Maina AN, Knobel DL, Cleaveland S, Laudisoit A, Wamburu K, et al. Molecular detection of Rickettsia felis and Candidatus Rickettsia asemboensis in fleas from human habitats, Asembo, Kenya. Vector Borne Zoonotic Dis. 2013;13:550-8. https://doi.org/10.1089/vbz.2012.1123

2. Maina AN, Jiang J, Luce-Fedrow A, St John HK, Farris CM, Richards AL. Worldwide presence and features of flea-borne
Rickettsia asembonensis. Front Vet Sci. 2019;5:334. https://doi.org/10.3389/fvets.2018.00334

3. Kho KL, Koh FX, Singh HKL, Zan HAM, Kukreja A, Ponnampalavanar S, et al. Case report: spotted fever group rickettsioses and murine typhus in a Malaysian teaching hospital. Am J Trop Med Hyg. 2016;95:765-8. https:/ / doi.org/ 10.4269/ajtmh.16-0199

4. Tay ST, Kho KL, Vythilingam I, Ooi CH, Lau YL. Investigation of possible rickettsial infection in patients with malaria. Trop Biomed. 2019;36:257-62.

5. Palacios-Salvatierra $\mathrm{R}$, Cáceres-Rey $\mathrm{O}$, Vásquez-Domínguez A, Mosquera-Visaloth $\mathrm{P}$, Anaya-Ramírez E. Rickettsial species in human cases with non-specific acute febrile syndrome in Peru [in Spanish]. Rev Peru Med Exp Salud Publica. 2018; 35:630-5. https:// doi.org/10.17843/rpmesp.2018.354.3646

6. Moonga LC, Hayashida K, Nakao R, Lisulo M, Kaneko C, Nakamura I, et al. Molecular detection of Rickettsia felis in dogs, rodents and cat fleas in Zambia. Parasit Vectors. 2019;12:168. https:// doi.org/10.1186/s13071-019-3435-6

7. Roucher C, Mediannikov O, Diatta G, Trape J-FF, Raoult D. A new Rickettsia species found in fleas collected from human dwellings and from domestic cats and dogs in Senegal. Vector Borne Zoonotic Dis. 2012;12:360-5. https://doi.org/10.1089/vbz.2011.0734

8. Zavala-Castro J, Zavala-Velázquez J, Walker D, Pérez-Osorio J, Peniche-Lara G. Severe human infection with Rickettsia felis associated with hepatitis in Yucatan, Mexico. Int J Med Microbiol. 2009;299:529-33. https:// doi.org/10.1016/j.ijmm.2009.03.002

9. Kato C, Chung I, Paddock C. Estimation of Rickettsia rickettsii copy number in the blood of patients with Rocky Mountain spotted fever suggests cyclic diurnal trends in bacteraemia. Clin Microbiol Infect. 2016;22:394-6. https:// doi.org/10.1016/j.cmi.2015.12.019

Address for correspondence: Kyoko Hayashida, Hokkaido University, International Institute for Zoonosis Control, Division of Collaboration and Education, Kita 20 Nishi 10, Kita-Ku, Sapporo 001-0020, Hokkaido, Japan; email: kyouko-h@czc.hokudai.ac.jp 